\title{
In-Situ Liquid Cell Electron Microscopy of the Solution Growth of Core-Shell Nanostructures
}

\author{
E. Sutter ${ }^{1}$, K. Jungjohann ${ }^{1}$, S. Bliznakov ${ }^{2}$, E. A. Stach ${ }^{1}$, A. Courty ${ }^{3}$, S. Wong ${ }^{4}$, C. Lewis ${ }^{4}$, and P. Sutter ${ }^{1}$ \\ 1. Center for Functional Nanomaterials, Brookhaven National Laboratory, Upton, New York, USA. \\ 2. Department of Chemistry, Brookhaven National Laboratory, Upton, New York, USA. \\ 3. Department of Chemistry, Université Pierre et Marie Curie, Paris, France. \\ 4. Department of Condensed Matter Physics and Materials Science, Brookhaven National Laboratory, \\ Upton, New York (USA).
}

Real-time electron microscopy observations of processes in wet environments, i.e., solutions, liquids, etc. have become possible only very recently with the development of electron beam transparent cells, in which a liquid layer is trapped between two ultrathin, electron-transparent membranes. Liquid-cell transmission electron microscopy (TEM) and scanning TEM (STEM) have attracted a lot of interest as they give direct insight into processes such as solution synthesis of nanostructures [1, 2], electrochemistry [3], biological phenomena, etc.

Here we present our in-situ liquid cell STEM observations of solution growth of metal nanoparticles and composite heterostructures of $\mathrm{Pd}$ and $\mathrm{Au}$. Bimetallic nanoparticles and composite heterostructures, such as core-shell nanoparticles, are of interest due to the promise of enhanced and tunable functional properties, for example increased catalytic activity and selectivity in a number of chemical reactions. Au-Pd bimetallic nanoparticles and core-shell nanostructures, in particular, demonstrate higher catalytic activity compared to monometallic catalysts in a variety of reactions [4].

We follow in real time and with high spatial resolution the homogeneous nucleation and growth of Pd, $\mathrm{Au}$, and $\mathrm{Au}-\mathrm{Pd}$ nanoparticles in dilute aqueous $\mathrm{Pd}$ and $\mathrm{Au}$ salt solutions, respectively. The growth exploits the fact that high-energy electron beams affect aqueous solutions containing metal ions or charged complexes through the generation of hydrated electrons and other radicals. Hydrated electrons $\left(\mathrm{e}_{\mathrm{aq}}{ }^{-}\right)$, for instance, are strong reducing agents that react with complex ions of most metals at a diffusioncontrolled rate. Their transfer from solution reduces the metal ions to zero-valent metal atoms or atom clusters. By incorporating nanoscale seeds of different materials, i.e. Au (figure 1) and $\mathrm{TiO}_{2}$ in the solution we investigate the templated growth of $\mathrm{Pd}, \mathrm{Au}$ or $\mathrm{Au}-\mathrm{Pd}$ on these seeds, and can thus follow the formation of complex nano-heterostructures. Real-time STEM observations allow us i) to follow the evolution of the growing material and the formation of heterostructures; ii) to directly identify different growth processes, i.e. growth in the solution, on the seed particle surface, or combinations of the two; and iii) to determine variations in growth rates at different surface sites on nanoparticles. The analysis of the $\mathrm{Pd}$ and $\mathrm{Au}$ incorporation at different distances from a focused electron beam provides a quantitative picture of the growth process, and shows that the growth is limited by the diffusion of $\mathrm{e}_{\mathrm{aq}}^{-}$in the solution [5] and by the mobility of the zero-valent metal monomers. We demonstrate that a judicious choice of the material and the morphology of the seeds provides control over the formation of a variety of heterostructures, such as core-shell nanoparticles with different shell morphologies, hollow-shells, etc. all of which promise enhanced and tunable functional properties, for example increased catalytic activity and selectivity in a number of chemical reactions. 


\section{References:}

[1] H M Zheng, et al, Science 324 (2009), p. 1309.

[2] J E Evans, et al, Nano Letters 11 (2011), p. 2809.

[3] M J Williamson, et al, Nature Mater. 2 (2003), p. 532.

[4] G J Hutchings, et al, Catalysis Today 138 (2008), p. 9.

[5] K L Jungjohann, et al, Nano Letters 13 (2013), p. 2964.

[6] Work performed at the Center for Functional Nanomaterials under the auspices of the U.S.

Department of Energy under contract No. DE-AC02-98CH10886.

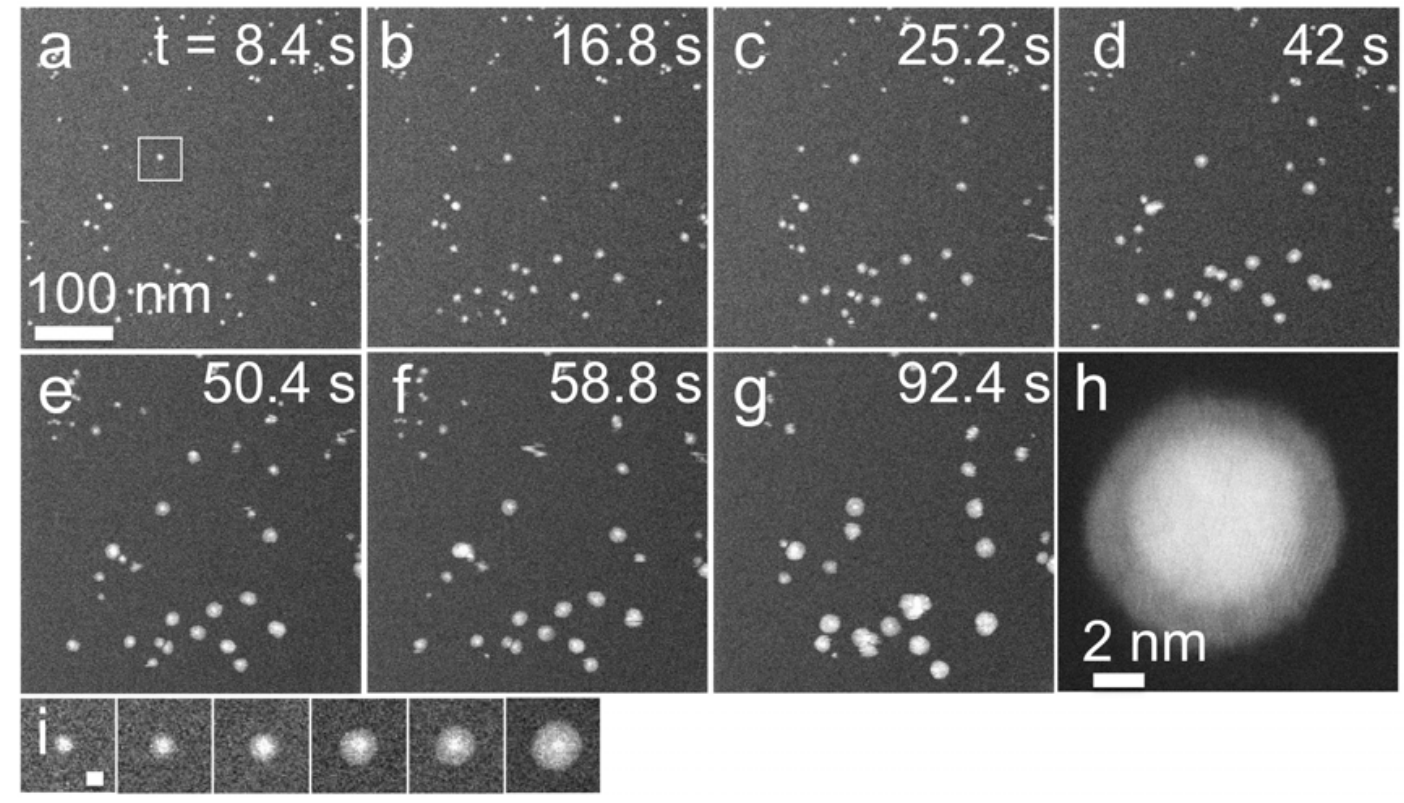

Figure 1:Pd growth on 5 nm Au nanoparticles seeds. a - Initial dark-field STEM image of an ensemble of $5 \mathrm{~nm}$ Au nanoparticles in $10 \mu \mathrm{M}$ aqueous $\mathrm{PdCl}_{2}$ solution. Electron dose per image: $150 \mathrm{e}^{-}$ $/ \AA^{2} \mathbf{a}-\mathbf{g}$ Time lapse series following the growth of Pd on the Au seeds over $92.4 \mathrm{~s}$. $\mathbf{h}-$ High-resolution Z-contrast image of a Au-Pd core-shell nanoparticle formed by Pd growth on the Au seeds. i - Image series showing the growth on an individual particle marked by a white frame in a (scale bar: $5 \mathrm{~nm}$ ). 